\title{
Excesso de peso em adolescentes: explorando potenciais fatores de risco
}

\author{
Overweight in adolescents: exploring potential risk factors
}

Jucemar Benedet ${ }^{1}$, Maria Alice A. de Assis², Maria Cristina M. Calvo ${ }^{3}$, Dalton Francisco de Andrade ${ }^{4}$

\section{RESUMO}

Objetivo: Estimar a prevalência de excesso de peso e identificar associações com fatores sociodemográficos, biológicos e de estilo de vida em adolescentes de Florianópolis, Santa Catarina.

Métodos: Estudo transversal, conduzido em 2007, com amostra probabilística de 1.590 escolares de 11 a 14 anos. Estimou-se a prevalência do excesso de peso, com base no índice de massa corpórea, por meio da referência brasileira e da International Obesity Task Force (IOTF). Análises multivariadas expressas como razão de chance foram usadas para identificar associações com fatores sociodemográficos, biológicos e de estilo de vida dos adolescentes.

Resultados: A prevalência de excesso de peso foi de $19,3 \%$ (24,5\% de meninos e 14,5\% de meninas), pela referência IOTF, e de 25,8\% (31,8\% de meninos e 20,5\% de meninas), pelo critério brasileiro. Entre os adolescentes do sexo masculino, o excesso de peso associou-se positivamente com a não realização de refeições estruturadas e com o deslocamento não ativo para a escola. Entre as meninas, o excesso de peso materno, a não realização de refeições estruturadas e diferenças interestágios de maturação sexual compuseram o elenco dos fatores associados ao excesso de peso.

Conclusões: A realização de refeições com alimentos de alto valor nutricional apresentou-se como um fator de proteção para o excesso de peso entre os adolescentes. Essa constatação reforça a necessidade de ações com vistas a mo- dificar comportamentos relacionados ao ambiente familiar, incentivando a incorporação de hábitos alimentares saudáveis e o lazer ativo.

Palavras-chave: adolescente; sobrepeso; obesidade; fatores de risco.

\section{ABSTRACT}

Objective: To estimate the prevalence of overweight and to identify associations with sociodemographic, biological and lifestyle factors in adolescents from Florianopolis, Santa Catarina, Brazil.

Methods: Cross-sectional study carried out in 2007 with a probabilistic sample of 1,590 schoolchildren aged from 11 to 14 years old. The prevalence of overweight, based on body mass index, was estimated by the Brazilian reference and the International Obesity Task Force (IOTF). Multivariate analysis expressed as odds ratios were used to identify associations with sociodemographic, biological and lifestyle factors among adolescents.

Results: The prevalence of overweight was 19.3\% (24.5\% in boys and $14.5 \%$ in girls) using the IOFT reference, and $25.8 \%$ (31.8\% in boys and 20.5\% in girls) by the Brazilian reference. Among male adolescents, overweight was positively associated with unbalanced food consumption and inactive commuting to school. Among girls, the factors associated with overweight were: mother's overweight,
Instituição: Universidade Federal de Santa Catarina (UFSC), Florianópolis, SC, Brasil

${ }^{1}$ Mestre pelo Programa de Pós-graduação em Nutrição do Centro de Ciências da Saúde da UFSC, Florianópolis, SC, Brasil

2Doutora em Engenharia de Produção pelo Programa de Pós-graduação em Engenharia de Produção do Centro Tecnológico da UFSC; Professora Associada do Programa de Pós-graduação em Nutrição do Centro de Ciências da Saúde da UFSC, Florianópolis, SC, Brasil

${ }^{3}$ Doutora em Engenharia de Produção pelo Programa de Pós-graduação em Engenharia de Produção do Centro Tecnológico da UFSC; Professora Associada do Programa de Pós-graduação em Saúde Coletiva do Centro de Ciências da Saúde da UFSC, Florianópolis, SC, Brasil

${ }^{4}$ Doutorado em Bioestatística pela University of North Carolina - MultiCampus University, Estados Unidos; Professor voluntário do Departamento de Engenharia de Produção da UFSC, Florianópolis, SC, Brasil
Endereço para correspondência:

Jucemar Benedet

UFSC, Campus Universitário - Trindade , s/n

CEP 88040-900 - Florianópolis/SC

E-mail: jucemarb@hotmail.com

Fonte financiadora: Conselho Nacional de Desenvolvimento Científico e Tecnológico (CNPq), processo n ${ }^{\circ}$ 402322/2005-3

Conflito de interesse: nada a declarar

Recebido em: 18/6/2012

Aprovado em: 12/11/2012 
unbalanced food consumption and discordance between stages for sexual maturity indicators.

Conclusions: The consumption of foods with high nutritional value was a protective factor against overweight among adolescents. This finding reinforces the importance of actions aimed at changing behaviors related to the family environment by encouraging the incorporation of healthy eating and active leisure time.

Key-words: adolescent; overweight; obesity; risk factors.

\section{Introdução}

Muitos estudos têm mostrado uma tendência no aumento do excesso de peso entre crianças e adolescentes nos Estados Unidos da América ${ }^{(1)}$, em países europeus ${ }^{(2)}$ e na América Latina ${ }^{(3)}$. No Brasil, a prevalência de sobrepeso entre crianças e adolescentes aumentou de 4,1 para 13,9\% entre 1974 e $1997^{(4)}$. Dados mais recentes, oriundos das Pesquisas de Orçamentos Familiares (POF) de 2002/20003 ${ }^{(5)}$ e 2008/2009 $9^{(6)}$, mostraram que a prevalência do excesso de peso na adolescência (dez a 19 anos) aumentou de 16,6 para $21,7 \%$ nos meninos e de 15,1 para $19,4 \%$ nas meninas ${ }^{(6)}$.

$\mathrm{O}$ excesso de gordura corporal durante o crescimento representa preditor importante de fatores de risco cardiovascular na vida adulta. A hipertensão arterial, o diabetes tipo II, as dislipidemias e a síndrome metabólica estão entre os principais problemas relacionados ao excesso de peso de crianças e adolescentes ${ }^{(7,8)}$. Além disso, o excesso de peso na adolescência acarreta efeitos psicológicos negativos, relacionados à formação da imagem corporal, depressão e problemas comportamentais ${ }^{(9)}$.

A obesidade é uma doença multifatorial e as interações entre os potenciais fatores determinantes são consideradas complexas. Há um consenso de que fatores ambientais, de estilo de vida e genéticos estão diretamente relacionados à composição corporal ${ }^{(10)}$. Na adolescência, o aumento do índice de massa corpórea (IMC) tem se mostrado associado principalmente aos comportamentos sedentários (relacionados a televisão, vídeo game e computador) e a um padrão alimentar pouco saudável, relacionado ao consumo de alimentos industrializados e com baixa densidade nutricional ${ }^{(11,12)}$.

Essas associações estão bem estabelecidas, porém outros fatores têm mostrado relação com o excesso de peso na adolescência e merecem ser mais bem investigados. Por exemplo, os estágios de maturação sexual, observando-se que, quando seu desenvolvimento e progressão não acontecem de forma uniforme, aumenta o risco de complicações relacionadas à obesidade, ao diabetes tipo II e à doença cardiovascular ${ }^{(13)}$. Da mesma forma, características sociodemográficas, aspectos relacionados ao ambiente familiar e à prática regular de atividades físicas têm se mostrado determinantes nesse processo. Portanto, o objetivo deste estudo foi estimar a prevalência e identificar potencias fatores de risco (sociodemográficos, comportamentais e biológicos) associados ao excesso de peso em adolescentes de 11 a 14 anos de Florianópolis, Santa Catarina.

\section{Método}

Este é um estudo transversal realizado em Florianópolis, de abril a outubro de 2007. As escolas participantes neste estudo foram escolhidas a partir de uma amostragem randomizada efetuada em uma pesquisa realizada em 2002 sobre a prevalência de sobrepeso e obesidade no município de Florianópolis ${ }^{(14)}$. Em 2002, o estudo utilizou um desenho de amostragem probabilística estratificado por conglomerados. As escolas foram agrupadas em quatro estratos, por área geográfica (centro/continente e praias) e por tipo de escola (pública e privada). Dentro de cada estrato, houve seleção aleatória das escolas com igual probabilidade. Do total de 87 escolas (33 privadas e 54 públicas), foram selecionadas 11 públicas e seis privadas. Nas escolas selecionadas, todas as classes e todas as crianças das primeiras às quartas séries foram convidadas a participar, mas somente as crianças com sete a dez anos de idade foram incluídas.

Em 2007, a amostra de escolares recrutada nessas escolas foi formada por dois grupos de escolares: 1) aqueles que, em 2002, tinham de sete a dez anos de idade e que, em 2007, tinham idade entre 11 a 14 anos; 2) escolares com idade entre 11 a 14 anos, randomicamente selecionados, com igual probabilidade, em cada uma das escolas. A inclusão do primeiro grupo teve a intenção de realizar seguimento dos alunos que participaram do estudo de prevalência em $2002^{(14)}$. Foram localizados, no censo da Secretaria Municipal de Educação, 1.100 escolares que fizeram parte da pesquisa em 2002, dos quais 735 participaram do presente estudo. O tamanho da amostra do segundo grupo foi calculado considerando-se a população escolar de 28.060 estudantes na faixa etária de 11 a 14 anos, prevalência de excesso de peso de $12,6 \%{ }^{(4)}$, com margem de erro de três pontos percentuais (bicaudal) e efeito de delineamento amostral de 1,5. Esse cálculo totalizou 700 adolescentes. Com as margens de erro para perdas de exames, a amostra estimada foi de 800 adolescentes de 11 a 14 anos. Finalmente, o processo de amostragem resultou em 
participação de 865 novos adolescentes e 735 adolescentes que haviam participado da pesquisa de 2002. Os dados de dez participantes foram excluídos, devido à ausência de dados antropométricos completos. Os critérios para a inclusão dos adolescentes foram baseados em três informações: 1) estar devidamente matriculado; 2) não apresentar nenhum tipo de deficiência física ou mental diagnosticada; 3) retornar o Termo de Consentimento Livre e Esclarecido devidamente assinado.

Os dados foram ponderados considerando-se a densidade populacional e a probabilidade de seleção em cada estrato da amostra. Os pesos da amostra foram usados para as estimativas de prevalência.

As informações coletadas incluíram medidas antropométricas: peso, estatura, dobras cutâneas e circunferências corporais. Para este estudo, utilizaram-se as medidas de peso e estatura. Os demais dados foram obtidos com a aplicação de três questionários: 1) questionário ilustrado para se obter dados sobre o tipo de transporte utilizado no deslocamento para a escola e os tipos de alimentos consumidos no dia anterior ao da entrevista; 2) questionário estruturado para avaliar as atividades físicas realizadas fora do ambiente escolar, atividades sedentárias e horas de sono; 3) questionário estruturado para se obter dados sociodemográficos dos pais (renda, peso, estatura e idade) e peso ao nascer do adolescente.

O termo de consentimento livre e esclarecido foi assinado pelos pais e o assentimento oral foi obtido dos adolescentes. A pesquisa foi aprovada pelo Comitê de Ética em Pesquisa com Seres Humanos da Universidade Federal de Santa Catarina (UFSC).

As medidas antropométricas foram realizadas de acordo com o protocolo de Lohman ${ }^{(15)}$. A equipe participou de estudo piloto e treinamento de padronização de medidas antropométricas para mensurar os erros intra e interavaliadores ${ }^{(16)}$. O peso corporal foi mensurado com os adolescentes usando roupas leves e sem calçado. Utilizou-se balança eletrônica com capacidade de $180 \mathrm{~kg}$ e escala de $100 \mathrm{~g}$. Para a medida de estatura, empregou-se estadiômetro com escala de $0,1 \mathrm{~mm}$. O IMC foi calculado com o peso $(\mathrm{kg})$ dividido pela altura (m) ao quadrado. $\mathrm{O}$ excesso de peso e a obesidade foram estimados usando-se os pontos de corte do IMC segundo a idade e o sexo da International Obesity Task Force $(\text { IOTF })^{(17)} \mathrm{e}$ do critério brasileiro ${ }^{(18)}$. Para as análises de associação entre excesso de peso e variáveis exploratórias, foram usados os pontos de corte da $\operatorname{IOTF}^{(17)}$.

O estado nutricional da mãe foi avaliado por meio do IMC com peso e altura autorreferidos. A classificação de excesso de peso $\left(\geq 25 \mathrm{~kg} / \mathrm{m}^{2}\right)$ seguiu a recomendação da Organização Mundial da Saúde (World Health Organization) ${ }^{(19)}$. A variável excesso de peso da mãe foi classificada de forma dicotômica (sim ou não).

Os adolescentes foram analisados segundo o tipo de escola que frequentavam — pública ou privada. Em relação às faixas etárias, os adolescentes foram classificados de acordo com as quatro idades que compõem a amostra (11 a 14 anos).

$\mathrm{O}$ peso ao nascer (em gramas) foi informado pelos pais ou responsáveis, que foram instruídos a consultar a caderneta de saúde. O peso ao nascer foi classificado em: baixo peso $(<2500 \mathrm{~g})$, peso normal $(\geq 2500-3999 \mathrm{~g})$ ou peso elevado $(\geq 4000 \mathrm{~g})^{(20)}$.

Foram utilizados os estágios de crescimento de pelos pubianos e de mamas ou genitais masculinos de acordo com o modelo proposto por Tanner ${ }^{(21)}$, em planilhas ilustradas. Os adolescentes autorreferiram individualmente os estágios de crescimento desses caracteres após receberem as orientações sobre o uso da planilha. Para as análises, as diferenças interestágios foram categorizadas em: estágios pareados (mesmo estágio para pelos pubianos e mamas/genitais); pelos pubianos adiantados em um ou dois estágios em relação às mamas/ genitais ou pelos pubianos atrasados em um ou dois estágios em relação às mamas/genitais.

As informações de consumo alimentar e transporte escolar foram avaliadas por meio do Questionário Alimentar do Dia Anterior (QUADA-3), previamente validado ${ }^{(22)}$. A variável referente à realização de refeições consideradas completas (estruturadas) teve como base a composição em alimentos de alta densidade nutricional. As três principais refeições (café da manhã, almoço e jantar) foram consideradas estruturadas quando apresentaram o consumo de pelo menos um alimento de cada um dos seguintes grupos: amido (arroz, feijão, pão, macarrão, bolacha água e sal); frutas e legumes (suco de frutas, frutas e legumes); e proteína animal (bife, frango, frutos do mar, ovo, leite, queijo, iogurte e achocolatado). Em relação aos lanches, utilizou-se o mesmo critério, porém era obrigatório o consumo de pelo menos um alimento de alta densidade nutricional, pertencente a qualquer grupo. Para as análises, os adolescentes foram classificados de forma dicotômica: realizar ou não três refeições estruturadas e dois lanches ao dia. O deslocamento para a escola foi classificado em ativo (caminhada e bicicleta) ou não ativo (carro, ônibus e carona de moto ou bicicleta).

Os adolescentes responderam a um questionário com perguntas sobre a prática de esportes além das aulas de 
Educação Física, comportamento sedentário (associado a televisão, vídeo game e computador) e horas de sono. O hábito de praticar esportes além das aulas de Educação Física foi classificado de forma dicotômica (com respostas sim ou não). Os dados referentes ao tempo dedicado a atividades sedentárias foram coletados em referência à média dos dias da semana e fim de semana e agrupados em uma única variável. O comportamento sedentário foi categorizado em: $0-3 \mathrm{~h}$, $>3 \mathrm{~h}$ e $\leq 6 \mathrm{~h}$ e $>6 \mathrm{~h}$ diárias. As horas de sono foram coletadas em relação à noite anterior e classificadas em duas categorias: $<8 \mathrm{~h}$ e $\geq 8 \mathrm{~h}$ de sono.

Os dados foram processados no programa Epi-Data 3.2, com dupla entrada. As análises foram realizadas com o software estatístico STATA 9.0 (STATA Corp. College Station, Texas, USA), corrigidas pelo delineamento complexo da amostra por meio da utilização do conjunto de comandos SVY do aplicativo, que incorpora as ponderações pertinentes a amostras complexas à estimação das variâncias. A prevalência de excesso de peso foi estimada para as categorias de cada variável independente. O nível de significância entre as proporções foi avaliado pelo teste do qui-quadrado para heterogeneidade ou para tendência linear. Para estimativas de risco, foram calculadas as razões de chance (RC), com intervalo de confiança (IC) de $95 \%$, por meio de regressão logística bivariada e multivariada. As variáveis com nível de significância $\leq 0,20$ foram incluídas na regressão logística multivariada pelo método backward. Valores de $p<0,05$ foram considerados estatisticamente significantes.

\section{Resultados}

Foram avaliados 1.590 escolares: 753 meninos e 837 meninas. Os escolares que se recusaram a participar ou para os quais os pais não forneceram o consentimento totalizaram 289 (148 do sexo masculino e 141 do feminino), porém foram substituídos por escolares previamente indicados para reposição no processo amostral.

$\mathrm{Na}$ Tabela 1, estão descritas as características antropométricas da amostra de acordo com o sexo. Observou-se evolução gradativa e esperada de peso e IMC em relação às

Tabela 1 - Peso, altura e índice de massa corpórea de adolescentes do sexo masculino e feminino

\begin{tabular}{|c|c|c|c|c|c|c|c|c|}
\hline \multirow[b]{2}{*}{ Idade (anos) } & \multicolumn{4}{|c|}{ Meninos } & \multicolumn{4}{|c|}{ Meninas } \\
\hline & 11 & 12 & 13 & 14 & 11 & 12 & 13 & 14 \\
\hline $\mathrm{n}$ & 177 & 214 & 205 & 157 & 189 & 225 & 241 & 182 \\
\hline \multicolumn{9}{|l|}{ Peso (kg) } \\
\hline Média & 42,9 & 46,2 & 53,3 & 58,2 & 41,8 & 46,7 & 50,2 & 54,2 \\
\hline Desvio padrão & 10,6 & 10,1 & 11,3 & 12,7 & 9,6 & 10,1 & 9,3 & 9,8 \\
\hline P25 & 34,8 & 38,5 & 45,4 & 49,8 & 35,7 & 40,5 & 43,9 & 47,4 \\
\hline P50 & 41,5 & 45,0 & 51,4 & 56,0 & 41,2 & 45,0 & 48,7 & 52,5 \\
\hline P75 & 47,6 & 52,7 & 60,3 & 65,0 & 46,9 & 50,9 & 55,2 & 59,7 \\
\hline \multicolumn{9}{|l|}{ Altura (cm) } \\
\hline Média & 148,7 & 153,4 & 161,2 & 166,4 & 149,6 & 154,3 & 158,8 & 160,2 \\
\hline Desvio padrão & 8,4 & 7,9 & 8,1 & 8,6 & 7,5 & 6,9 & 6,9 & 6,8 \\
\hline $\mathrm{P} 25$ & 143,3 & 148,1 & 156,5 & 161,7 & 144,6 & 150,5 & 154,4 & 155,1 \\
\hline P50 & 147,6 & 153,1 & 160,8 & 166,5 & 150,0 & 154,3 & 158,7 & 160,3 \\
\hline P75 & 153,6 & 158,5 & 167,2 & 172,1 & 155,2 & 158,5 & 163,4 & 164,7 \\
\hline \multicolumn{9}{|l|}{ IMC $\left(\mathrm{kg} / \mathrm{m}^{2}\right)$} \\
\hline Média & 19,2 & 19,5 & 20,4 & 20,9 & 18,5 & 19,5 & 19,9 & 21,0 \\
\hline Desvio padrão & 3,5 & 3,1 & 3,3 & 3,6 & 3,2 & 3,5 & 3,3 & 3,2 \\
\hline P25 & 16,7 & 17,4 & 17,9 & 18,7 & 16,3 & 17,2 & 17,8 & 18,8 \\
\hline P50 & 18,6 & 18,7 & 19,7 & 20,0 & 18,0 & 18,8 & 19,4 & 20,5 \\
\hline P75 & 21,1 & 21,2 & 22,7 & 22,0 & 19,9 & 21,2 & 21,3 & 22,8 \\
\hline
\end{tabular}

P: percentil. 
faixas etárias investigadas, em ambos os sexos. A mesma tendência foi observada em relação à altura dos meninos. Para as meninas com 13 e 14 anos, observou-se tendência para estabilização da altura.

A Tabela 2 apresenta a prevalência de obesidade e excesso de peso, por idade e sexo, segundo as referências $\operatorname{IOTF}^{(17)} \mathrm{e}$ Brasil ${ }^{(18)}$. A prevalência de obesidade e excesso de peso foi maior entre os meninos, independentemente do critério diagnóstico utilizado. Observou-se também comportamento não linear das prevalências de obesidade e excesso de peso entre as faixas etárias avaliadas, em ambos os sexos. Para todas as faixas etárias avaliadas, as prevalências foram maiores para as curvas de referência do critério brasileiro ${ }^{(18)}$, independentemente do sexo. A inclusão do grupo de seguimento não introduziu viés nas estimativas, tendo em vista que não houve diferença estatisticamente significante entre os dois grupos de escolares quanto à prevalência de excesso de peso, independentemente do critério diagnóstico utilizado: por exemplo, grupo de seguimento - 18,9\% (IC95\% 16,1-21,7) versus grupo de novos adolescentes 21\% (IC95\% 18,2-21,7; critério IOTF.

A Tabela 3 apresenta as prevalências de excesso de peso e razões de chance bruta e ajustada para os meninos. Adolescentes que estudavam em escola privada apresentaram maior chance para excesso de peso; porém, essas diferenças não se mantiveram estatisticamente significantes após o ajuste para outras variáveis. Adolescentes cujas mães foram classificadas como portadoras de excesso

Tabela 2 - Prevalência de excesso de peso e obesidade dos adolescentes estudados segundo as curvas de referência do índice de massa corpórea da International Obesity Task Force ${ }^{17}$ e Brasil - $2006^{18}$

\begin{tabular}{|c|c|c|c|c|c|c|c|}
\hline \multirow{2}{*}{ Idade } & \multirow{2}{*}{$\mathbf{n}$} & \multicolumn{3}{|c|}{ IOTF } & \multicolumn{3}{|c|}{ Brasil $^{*}$} \\
\hline & & \multirow[t]{2}{*}{ Média } & \multicolumn{2}{|c|}{ IC95\% } & \multirow[t]{2}{*}{ Média } & \multicolumn{2}{|c|}{ IC95\% } \\
\hline \multicolumn{6}{|c|}{ Meninos - Obesidade } & & \\
\hline 11 & 177 & 7,4 & 1,6 & 13,1 & 7,4 & 1,6 & 13,1 \\
\hline 12 & 214 & 4,3 & 1,5 & 7,1 & 3,4 & 0,1 & 6,6 \\
\hline 13 & 205 & 3,5 & 1,0 & 6,0 & 3,5 & 1,0 & 6,0 \\
\hline 14 & 157 & 5,3 & 2,9 & 7,7 & 7,6 & 2,4 & 12,8 \\
\hline Total & 753 & 5,0 & 2,9 & 7,0 & 5,2 & 3,0 & 7,3 \\
\hline \multicolumn{8}{|c|}{ Meninos - Excesso de peso } \\
\hline 11 & 177 & 28,3 & 16,5 & 40,1 & 33,8 & 20,8 & 46,8 \\
\hline 12 & 214 & 21,6 & 15,8 & 27,3 & 28,9 & 21,8 & 35,9 \\
\hline 13 & 205 & 24,9 & 19,3 & 30,5 & 35,8 & 29,9 & 41,8 \\
\hline 14 & 157 & 23,9 & 16,1 & 30,7 & 28,0 & 20,8 & 35,1 \\
\hline Total & 753 & 24,5 & 20,4 & 28,6 & 31,7 & 26,1 & 37,3 \\
\hline \multicolumn{8}{|c|}{ Meninas - Obesidade } \\
\hline 11 & 189 & 3,4 & 1,8 & 8,8 & 4,8 & 1,3 & 11,0 \\
\hline 12 & 225 & 3,5 & 0,3 & 7,4 & 5,0 & 0,1 & 9,1 \\
\hline 13 & 241 & 2,4 & 0,8 & 3,9 & 3,0 & 0,7 & 5,3 \\
\hline 14 & 182 & 2,0 & 0,3 & 3,7 & 3,6 & 0,9 & 6,3 \\
\hline Total & 837 & 2,8 & 1,1 & 4,6 & 4,1 & 1,4 & 6,7 \\
\hline \multicolumn{8}{|c|}{ Meninas - Excesso de peso } \\
\hline 11 & 189 & 13,5 & 4,6 & 22,4 & 18,8 & 7,5 & 30,1 \\
\hline 12 & 225 & 15,8 & 7,9 & 23,0 & 25,9 & 20,9 & 30,9 \\
\hline 13 & 241 & 10,9 & 7,7 & 14,0 & 15,6 & 12,3 & 18,9 \\
\hline 14 & 182 & 18,9 & 14,0 & 23,9 & 21,9 & 17,0 & 26,8 \\
\hline Total & 837 & 14,5 & 10,2 & 18,8 & 20,4 & 16,9 & 24,0 \\
\hline
\end{tabular}

IOTF: International Obesity Task Force ${ }^{(17) ;}$ "Conde e Monteiro, 2006(18); IC95\%: intervalo de confiança de $95 \%$. 
de peso apresentaram prevalências 58\% maiores para excesso de peso. Nas análises bivariadas, o peso ao nascer apresentou relação positiva e com gradiente linear em relação ao excesso de peso dos adolescentes; no entanto, essa associação não se mostrou estatisticamente significante no modelo final.

Entre as variáveis comportamentais, adolescentes que relataram maior tempo dedicado às atividades sedentárias apresentaram maiores prevalências de excesso de peso nas análises brutas. As horas de sono foram inversamente proporcionais à prevalência de excesso de peso, porém sem diferenças significantes. O uso de formas não ativas de deslocamento para a escola e a não realização de refeições estruturadas mostraram ser fatores associados ao excesso de peso, mesmo após ajuste para outros preditores.

A Tabela 4 apresenta as prevalências de excesso de peso e razões de chance bruta e ajustada para as meninas. Adolescentes que estudavam em escolas privadas

Tabela 3 - Análise bruta e ajustada para excesso de peso e fatores associados entre adolescentes do sexo masculino

\begin{tabular}{|c|c|c|c|c|c|c|}
\hline Variáveis & $\mathbf{n}$ & $\begin{array}{l}\text { Excesso de } \\
\text { peso }(\%)\end{array}$ & $\begin{array}{l}\text { RC bruta } \\
\text { IC95\% }\end{array}$ & Valor $p$ & $\begin{array}{c}\text { RC ajustada } \\
\text { IC } 95 \%\end{array}$ & Valor $p$ \\
\hline Escola & & & & 0,028 & & 0,074 \\
\hline Pública & 192 & 22,03 & 1,00 & & 1,00 & \\
\hline Privada & 561 & 30,12 & $1,52(1,05-2,20)$ & & $1,43(0,95-2,14)$ & \\
\hline Idade (anos completos) & & & & 0,620 & & \\
\hline 11 & 177 & 28,03 & 1,00 & & & \\
\hline 12 & 214 & 21,62 & $0,69(0,38-1,25)$ & & & \\
\hline 13 & 205 & 24,95 & $0,84(0,45-1,56)$ & & & \\
\hline 14 & 157 & 23,93 & $0,79(0,41-1,52)$ & & & \\
\hline IMC da mãe $\geq 25 \mathrm{~kg} / \mathrm{m}^{2 *}$ & & & & 0,129 & & 0,070 \\
\hline Não & 448 & 21,70 & 1,00 & & 1,00 & \\
\hline Sim & 252 & 30,47 & $1,58(0,85-2,92)$ & & $1,77(0,94-3,33)$ & \\
\hline Peso ao nascer (g) & & & & 0,049 & & 0,107 \\
\hline$<2500$ & 92 & 19,59 & 1,00 & & 1,00 & \\
\hline 2500-3999 & 577 & 24,38 & $1,32(0,63-2,76)$ & & $1,46(0,62-3,42)$ & \\
\hline$\geq 4000$ & 83 & 31,68 & $1,90(0,86-4,16)$ & & $1,94(0,80-4,66)$ & \\
\hline Maturação sexual & & & & 0,904 & & \\
\hline Estágios pareados & 447 & 23,85 & 1,00 & & & \\
\hline Pelos pubianos adiantados & 138 & 27,36 & $1,20(0,60-2,37)$ & & & \\
\hline Pelos pubianos atrasados & 161 & 22,68 & $0,93(0,66-1,31)$ & & & \\
\hline Refeições estruturadas & & & & 0,006 & & 0,024 \\
\hline Não & 289 & 31,72 & 1,00 & & 1,00 & \\
\hline Sim & 464 & 20,03 & $0,53(0,36-0,80)$ & & $0,59(0,39-0,92)$ & \\
\hline Esporte fora da escola & & & & 0,838 & & \\
\hline Sim & 566 & 24,80 & 1,00 & & & \\
\hline Não & 171 & 23,87 & $0,95(0,55-1,62)$ & & & \\
\hline Deslocamento para a escola & & & & 0,021 & & 0,017 \\
\hline Ativo & 361 & 21,34 & 1,00 & & 1,00 & \\
\hline Não ativo & 390 & 27,68 & $1,41(1,06-1,86)$ & & $1,36(1,06-1,73)$ & \\
\hline Atividades sedentárias (h/dia) & & & & 0,105 & & 0,476 \\
\hline $0-3 \mathrm{~h}$ & 213 & 23,12 & 1,00 & & & \\
\hline$>3$ e $\leq 6 h$ & 361 & 24,37 & $1,07(0,63-1,79)$ & & $1,02(0,60-1,74)$ & \\
\hline$>6$ a $10 \mathrm{~h}$ & 170 & 26,46 & $1,19(0,66-2,14)$ & & $1,26(0,67,2,33)$ & \\
\hline Horas de sono/dia & & & & 0,345 & & \\
\hline$<8$ & 294 & 26,78 & 1,00 & & & \\
\hline$\geq 8$ & 440 & 22,98 & $0,81(0,51-1,28)$ & & & \\
\hline
\end{tabular}

*Taxa de resposta de 93\%; RC: razão de chances; IC95\%: intervalo de confiança de 95\%; IMC: índice de massa corpórea. 
apresentaram prevalências significativamente menores de excesso de peso em relação às que estudavam em escola pública. Nas análises ajustadas, esta tendência também foi observada, porém com IC limítrofe (OR 0,53; IC95\% 0,29$1,00)$. A prevalência de excesso de peso aumentou de forma significativa com o excesso de peso materno, independentemente de outras variáveis. A variável diferenças interestágios de maturação sexual duplicou a chance para excesso de peso quando as meninas apresentaram as mamas em estágios mais desenvolvidos em relação aos pêlos pubianos. No modelo ajustado, entre as variáveis comportamentais, somente a realização de refeições estruturadas mostrou ser fator de proteção para o excesso de peso. Adicionalmente, cabe destacar, na análise bruta, a associação positiva e com gradiente linear entre os comportamentos sedentários e o excesso de peso, assim como as menores prevalências observadas entre as adolescentes que relataram dormir mais de 8 h e praticar esportes fora da escola.

Tabela 4 - Análise bruta e ajustada para excesso de peso e fatores associados entre adolescentes do sexo feminino

\begin{tabular}{|c|c|c|c|c|c|c|}
\hline Variáveis & $\mathbf{n}$ & $\begin{array}{c}\text { Excesso de } \\
\text { peso }(\%)\end{array}$ & $\begin{array}{c}\text { RC bruta } \\
\text { IC } 95 \%\end{array}$ & Valor $p$ & $\begin{array}{c}\text { RC ajustada } \\
\text { IC } 95 \% \\
\end{array}$ & Valor $p$ \\
\hline Escola & & & & 0,021 & & 0,053 \\
\hline Pública & 616 & 17,24 & 1,00 & & & \\
\hline Privada & 221 & 8,78 & $0,45(0,24-0,86)$ & & $0,54(0,29-1,00)$ & \\
\hline Idade (anos completos) & & & & 0,565 & & \\
\hline 11 & 189 & 13,51 & 1,00 & & & \\
\hline 12 & 225 & 15,89 & $1,29(0,62-2,32)$ & & & \\
\hline 13 & 241 & 10,91 & $0,78(0,38-1,60)$ & & & \\
\hline 14 & 182 & 19,00 & $1,50(0,71-3,16)$ & & & \\
\hline IMC da mãe $\geq 25 \mathrm{~kg} / \mathrm{m}^{2 *}$ & & & & 0,000 & & $<0,001$ \\
\hline Não & 510 & 8,65 & 1,00 & & & \\
\hline $\operatorname{Sim}$ & 288 & 26,32 & $3,77(2,24-6,32)$ & & $3,37(2,10-5,41)$ & \\
\hline Peso ao nascer (g) & & & & 0,527 & & \\
\hline$<2500$ & 98 & 15,02 & 1,00 & & & \\
\hline 2500-3999 & 693 & 14,18 & $0,93(0,48-1,79)$ & & & \\
\hline$\geq 4000$ & 43 & 20,67 & $1,47(0,53-4,04)$ & & & \\
\hline Maturação sexual & & & & 0,020 & & 0,022 \\
\hline Estágios pareados & 447 & 13,14 & 1,00 & & & \\
\hline Pelos pubianos adiantados & 201 & 8,76 & $0,63(0,36-1,10)$ & & $0,67(0,34-1,32)$ & \\
\hline Pelos pubianos atrasados & 179 & 25,63 & $2,27(1,29-4,01)$ & & $2,39(1,24-4,60)$ & \\
\hline Refeições estruturadas & & & & 0,001 & & 0,004 \\
\hline Não & 384 & 21,38 & 1,00 & & 1,00 & \\
\hline Sim & 453 & 9,07 & $0,36(0,22-0,60)$ & & $0,41(0,24-0,69)$ & \\
\hline Esporte fora da escola & & & & 0,218 & & \\
\hline $\operatorname{Sim}$ & 424 & 13,52 & 1,00 & & & \\
\hline Não & 404 & 15,88 & $1,20(0,87-1,65)$ & & & \\
\hline Deslocamento para a escola & & & & 0,825 & & \\
\hline Ativo & 378 & 14,23 & 1,00 & & & \\
\hline Não ativo & 457 & 14,90 & $1,05(0,62-1,77)$ & & & \\
\hline Atividades sedentárias ( $\mathrm{h} / \mathrm{dia}$ ) & & & & 0,047 & & 0,204 \\
\hline $0-3 h$ & 244 & 12,08 & 1,00 & & & \\
\hline$>3$ e $\leq 6 h$ & 434 & 14,71 & $1,25(0,68-2,30)$ & & $1,27(0,66-2,44)$ & \\
\hline$>6$ a $10 h$ & 154 & 18,71 & $1,67(0,78-3,58)$ & & $1,56(0,76-3,21)$ & \\
\hline Horas de sono/dia & & & & 0,314 & & \\
\hline$<8$ & 305 & 16,22 & 1,00 & & & \\
\hline$\geq 8$ & 527 & 13,67 & $0,81(0,53-1,24)$ & & & \\
\hline
\end{tabular}

*Taxa de resposta de 95,2\%; RC: razão de chances; IC95\%: intervalo de confiança de 95\%; IMC: índice de massa corpórea. 


\section{Discussão}

O estudo encontrou diferentes fatores associados ao excesso de peso para meninos e meninas, confirmando sua natureza multicausal e com características específicas para cada sexo. A utilização de delineamento transversal confere ao estudo as limitações inerentes à causalidade reversa, principalmente relacionada às variáveis comportamentais. Algumas informações, tais como consumo alimentar, horas de sono e horas de atividade sedentária, foram recordadas pelos adolescentes e estão sujeitas ao viés de memória e de comportamentos socialmente desejáveis. Nesse mesmo sentido, as informações autorreferidas para determinação do IMC da mãe e estadiamento puberal dos adolescentes podem estar sujeitas a erros de interpretação. No entanto, estudos demonstraram elevado grau de correlação entre medidas de estatura e massa corporal referidas e auferidas em adultos $(r=0,85-0,97)^{(23,24)}$. Em relação à maturação sexual, estudos mostram correlação moderada a alta $(r=0,60-0,71)$ para as medidas de estadiamento puberal provenientes da autoavaliação e daquela realizada por profissional especializado ${ }^{(25,26)}$.

Observou-se alta prevalência de excesso de peso, independentemente do critério de IMC utilizado, principalmente entre os meninos. A prevalência de sobrepeso/excesso de peso estimada com o uso da referência brasileira foi maior do que com a da IOTF (25,8 versus 19,3\%). As diferenças são esperadas, pois os critérios diferem entre si pelo país de origem e particularidades étnicas associadas à grande variabilidade dos valores de IMC na infância ${ }^{(27)}$.

As análises revelaram que estudar em escola pública aumenta as chances para o excesso de peso em adolescentes do sexo feminino, enquanto que, para os meninos, esse efeito é inverso. Ainda que, para ambos os sexos, as diferenças não tenham se mostrado significativas no modelo final, entre as meninas essa tendência já havia sido observada em outro estudo na cidade de Florianópolis para a mesma faixa etária ${ }^{(28)}$ Comportamentos distintos de meninos e meninas relacionados à estética corporal, à realização de dietas para controle de peso e ao consumo de alimentos com baixa densidade energética, notoriamente mais presentes entre as meninas e em estratos sociais mais elevados, são hipóteses plausíveis para tal realidade.

A associação observada entre o estado nutricional materno e o excesso de peso dos adolescentes são similares aos achados de estudos conduzidos no Brasil ${ }^{(29)}$ e em outros países ${ }^{(30,31)}$. Os hábitos de vida caracterizados pelo ambiente obesogênico, somados à herança genética, são apontados como importantes agentes da obesidade. No presente estudo, o excesso de peso materno aumentou as chances para o excesso de peso, independenteente do sexo e do tipo de escola, ainda que, no caso dos meninos, não tenha se sustentado no modelo final. Essa constatação proporciona bons indicativos para políticas publicas de prevenção, as quais devem estar vinculadas ao núcleo familiar independentemente das condições sociodemográficas.

A maturação sexual tem estreita relação com os níveis de gordura corporal, principalmente entre as meninas. A discordância entre os estágios de maturação sexual pode oferecer indicativos do crescimento físico, funções reprodutivas e IMC ${ }^{(13)}$. Neste estudo, as diferenças no pareamento dos estágios e o excesso de peso foram significativos entre as meninas. Quando o desenvolvimento das mamas mostrou-se adiantado em relação aos pelos pubianos em um ou dois estágios, o risco para sobrepeso mais que dobrou (OR 2,39; IC95\% 1,24-4,60). Os resultados foram similares aos encontrados em estudo com adolescentes americanas ${ }^{(13)}$. As discordâncias entre estágios podem ser o reflexo de alterações no equilíbrio hormonal, sendo plausível supor que afetem o crescimento físico, o processo de maturação sexual e a composição corporal.

Meninos e meninas apresentaram comportamentos similares em relação às características das refeições realizadas e o excesso de peso. Comer de forma estruturada nas principais refeições do dia mostrou efeito protetor para o excesso de peso. Por outro lado, a não inclusão de alimentos saudáveis e tradicionais nas refeições pode indicar que os adolescentes estejam consumindo, em maior quantidade, alimentos com alta densidade energética ou mesmo diminuindo o número de refeições realizadas durante o dia. É possível que a não realização de refeições consideradas estruturadas faça parte de estratégia para redução do peso corporal, visto que dietas restritivas e com pouca variação de alimentos são costumeiramente empregadas na tentativa de emagrecer ${ }^{(32)}$.

O deslocamento para escola e sua relação com o excesso de peso ainda é pouco investigado no Brasil. A maioria dos estudos explora a atividade física relacionada ao lazer, porém é preciso considerar que os deslocamentos juntamente com o lazer são os contextos que incorporam quase a totalidade das atividades físicas de crianças e adolescentes. Neste estudo, as formas de transporte não ativo aumentaram as chances para o excesso de peso em meninos, independentemente de outros fatores investigados. Sem dúvida, as questões que envolvem a adoção de formas de deslocamento ativas pelos adolescentes vão além das motivações pessoais e incorporam 
questões de segurança e acessibilidade. Políticas públicas devem ser incentivadas nesse sentido.

Para ambos os sexos, o comportamento sedentário aumentou as chances de excesso de peso, ainda que, nos modelos finais, essa associação não tenha se sustentado. Há indícios de que uma das possíveis consequências da exposição prolongada às formas de lazer sedentário esteja relacionada ao menor consumo de frutas e hortaliças e à distribuição inadequada de macronutrientes ${ }^{(33)}$. Da mesma forma, especula-se sobre a provável diminuição dos níveis de atividade física com o advento das formas eletrônicas de lazer. Uma metanálise mostrou associação negativa entre assistir televisão, usar computadores e praticar atividade física ${ }^{(34)}$. No entanto, essas hipóteses precisam ser mais bem investigadas, para que se possam elucidar tais associações e atribuir um possível efeito de causalidade.

É importante destacar que determinados padrões de comportamento vão além das questões sociodemográficas e se constituem pontos chave para programas de intervenção. Os hábitos alimentares, por exemplo, são reflexos da

\section{Referências bibliográficas}

1. Lobstein T, Jackson-Leach R. Child overweight and obesity in the USA: prevalence rates according to IOTF definitions. Int J Pediatr Obes 2007;2:62-4.

2. Jackson-Leach R, Lobstein T. Estimated burden of paediatric obesity and comorbidities in Europe. Part 1. The increase in the prevalence of child obesity in Europe is itself increasing. Int J Pediatr Obes 2006;1:26-32.

3. Amigo $\mathrm{H}$. Obesidad en el niño en América Latina: situación, criterios de diagnóstico y desafíos. Cad Saude Publica 2003;19(Suppl 1):S163-70.

4. Wang Y, Monteiro C, Popkin BM. Trends of obesity and underweight in older children and adolescents in the United States, Brazil, China, and Russia. Am J Clin Nutr 2002;75:971-7.

5. Instituto Brasileiro de Geografia e Estatística [homepage on the Internet]. Pesquisa de orçamentos familiares 2002-2003 (POF). Antropometria e análise do estado nutricional de crianças e adolescentes no Brasil [cited 2008 Jan 10]. Available from: http://www.ibge.gov.br/home/estatistica/populacao/ condicaodevida/pof/2003medidas/default.shtm

6. Instituto Brasileiro de Geografia e Estatística [homepage on the Internet]. Pesquisa de orçamentos familiares 2008-2009 (POF). Antropometria e estado nutricional de crianças, adolescentes e adultos no Brasil [cited 2011 Jan 5]. Available from: http://www.ibge.gov.br/home/estatistica/populacao/ condicaodevida/pof/2008_2009_encaa/default.shtm

7. Lobstein T, Baur L, Uauy R; IASO International Obesity Task Force. Obesity in children and young people: a crisis in public health. Obes Rev 2004;5 (Suppl 1):S4-104.

8. Berenson GS, Agirbasli M, Nguyen QM, Chen W, Srinivasan SR. Glycemic status, metabolic syndrome, and cardiovascular risk in children. Med Clin North Am 2011;95:409-17.

9. Puder JJ, Munsch S. Psychological correlates of childhood obesity. Int J Obes (Lond) 2010;34 (Suppl 2):S37-43. cultura familiar e podem interferir diretamente no estado nutricional em qualquer fase da vida. Assim, políticas de prevenção devem ser conduzidas no intuito de modificar comportamentos relacionados ao ambiente familiar, em todos os estratos sociais. Devem-se planejar ações com vistas a incentivar o lazer ativo em detrimento do uso de computadores e televisão, juntamente com a incorporação de hábitos alimentares saudáveis, o que inclui refeições com alimentos de alta densidade nutricional.

Por fim, os achados indicam que o excesso de peso associou-se a diferentes fatores entre meninos e meninas, com exceção da não realização de refeições estruturadas, cujo efeito independeu de variáveis sociodemográficas. Entre as meninas, a relação com os fatores endócrinos vinculados aos estágios de maturação sexual, juntamente com o estado nutricional materno, foram fatores que agregaram maior chance para o excesso de peso. Por outro lado, nos meninos, a associação do excesso de peso com o deslocamento não ativo para a escola e com o fato de estudarem na rede privada indicam um cenário a ser mais bem observado em novos estudos.
10. Hebebrand J, Hinney A. Environmental and genetic risk factors in obesity Child Adolesc Psychiatr Clin N Am 2009;18:83-94.

11. Hills AP, King NA, Armstrong TP. The contribution of physical activity and sedentary behaviours to the growth and development of children and adolescents: implications for overweight and obesity. Sports Med 2007;37:533-45.

12. Moreno LA, Rodríguez G. Dietary risk factors for development of childhood obesity. Curr Opin Clin Nutr Metab Care 2007;10:336-41.

13. Schubert CM, Chumlea WC, Kulin HE, Lee PA, Himes JH, Sun SS. Concordant and discordant sexual maturation among U.S. children in relation to body weight and BMI. J Adolesc Health 2005;37:356-62.

14. de Assis MA, Rolland-Cachera MF, Grosseman S, de Vasconcelos FA, Luna $\mathrm{ME}$, Calvo MC et al. Obesity, overweight and thinness in schoolchildren of the city of Florianópolis, Southern Brazil. Eur J Clin Nutr 2005;59:1015-21.

15. Lohman TG, Roche AF, Martorell R. Anthropometric standardization reference manual. Champaign (IL): Human Kinetics; 1988.

16. Frainer DE, Adami F, Vasconcelos F de A, Assis MA, Calvo MC, Kerpel R. Standardization and reliability of anthropometric measurements for population surveys. Arch Latinoam Nutr 2007;57:335-42.

17. Cole TJ, Bellizzi MC, Flegal KM, Dietz WH. Establishing a standard definition for child overweight and obesity worldwide: international survey. BMJ 2000;320:1240-3.

18. Conde WL, Monteiro CA. Body mass index cutoff points for evaluation of nutritional status in Brazilian children and adolescents. J Pediatr (Rio J) 2006; 82:266-72.

19. World Health Organization. Obesity: preventing and managing the globa epidemic. Report of a WHO consultation on obesity. Geneva: WHO; 1998.

20. World Health Organization. Physical status: the use and interpretation of anthropometry. Report of a WHO expert committee. Geneva: WHO; 1995. 
21. Tanner JM. Growth at adolescence. Oxford: Blackwell Scientific Publications; 1962.

22. Assis MA, Benedet J, Kerpel R, Vasconcelos FA, Pietro PF, Kupek E. Validação da terceira versão do Questionário Alimentar do Dia Anterior (QUADA-3) para escolares de 6 a 11 anos. Cad Saude Publica 2009;25:1816-26.

23. Fonseca MJ, Faerstein E, Chor D, Lopes CS. Validade de peso e estatura informados e índice de massa corporal: estudo pró-saúde. Rev Saude Publica 2004;38:392-8.

24. Silveira EA, Araújo CL, Gigante DP, Barros AJ, Lima MS. Validação do peso e altura referidos para o diagnóstico do estado nutricional em uma população de adultos no sul do Brasil. Cad Saude Publica 2005;21:235-45.

25. Matsudo SM, Matsudo VK. Validade da auto-avaliação na determinação da maturação sexual. Rev Bras Ciênc Mov 1991;5:18-35.

26. Matsudo SM, Matsudo VK. Self-assessment and physician assessment of sexual maturation in Brazilian boys and girls: concordance and reproducibility. Am J Hum Biol 1994;6:451-5.

27. Tomkins A. Measuring obesity in children: what standards to use? J Pediatr (Rio J) 2006;82:246-8.
28. Silva KS, Pelegrini A, Hoefelmann LP, Vasques DG, Lopes AS. Prevalência de excesso de peso corporal em escolas públicas e privadas da cidade de Florianópolis, SC. Arq Bras Endocrinol Metab 2008;52:574-5.

29. Giugliano R, Carneiro EC. Fatores associados à obesidade em escolares. J Pediatr (Rio J) 2004;80:17-22.

30. Moraes SA, Rosas JB, Mondini L, Freitas IC. Prevalência de sobrepeso e obesidade e fatores associados em escolares de área urbana de Chilpancingo, Guerrero, Mexico, 2004. Cad Saude Publica 2006;22:1289-301.

31. Ramos E, Barros H. Family and school determinants of overweight in 13-yearold Portuguese adolescents. Acta Paediatr 2007;96:281-6.

32. Bernardi F, Cichelero C, Vitolo MR. Comportamento de restrição alimentar e obesidade. Rev Nutr 2005;18:85-93.

33. Ortega RM, Requejo AM, Andrés P, López-Sobaler AM, Redondo MR, González-Fernández M. Influence of the time spent watching television on the dietary habits, energy intake and nutrient intake of a group of Spanish adolescents. Nutr Res 1996;16:1467-70.

34. Marshall SJ, Biddle SJ, Gorely T, Cameron N, Murdey I. Relationships between media use, body fatness and physical activity in children and youth: a metaanalysis. Int J Obes Relat Metab Disord 2004;28:1238-46. 